- molecular

CANCER

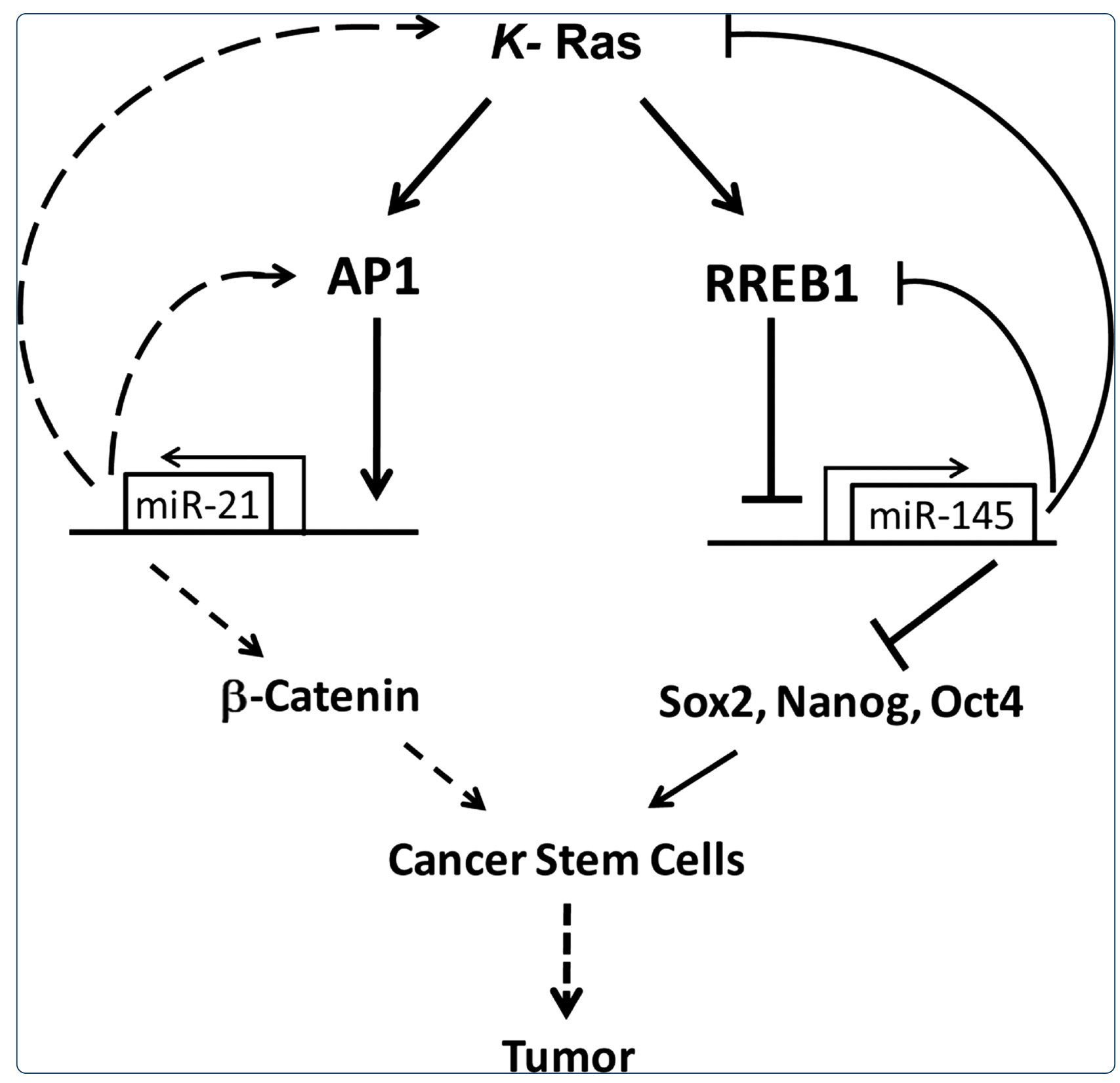

miR-21 and miR-145 cooperation in regulation of colon cancer stem cells

Yu et al.

( ) Biomed Central 


\title{
miR-21 and miR-145 cooperation in regulation of colon cancer stem cells
}

\author{
Yingjie $\mathrm{Yu}^{1,3^{*}}$, Pratima Nangia-Makker ${ }^{1,2,3}$, Lulu Farhana ${ }^{1,3}$, Sindhu G. Rajendra ${ }^{1}$, Edi Levi ${ }^{1}$
} and Adhip PN Majumdar ${ }^{1,2,3^{*}}$

\begin{abstract}
Background: Acquired drug resistance is one of the major reasons for failing cancer therapies. Although the reasons are not fully understood, they may be related to the presence of cancer stem cells (CSCs). We have reported that chemo-resistant (CR) colon cancer cells, highly enriched in CSCs, exhibit a marked up-regulation of miR-21 and that down-regulation of this miR renders the $C R$ cells more susceptible to therapeutic regimens. However, the underlying molecular mechanism is poorly understood. The aim of this investigation is to unravel this mechanism.
\end{abstract}

Methods: The levels of miR-145 and miR-21 were manipulated by transfection of mature, antago-miRs or pCMV/miR-145 expression plasmid. Quantitative RT-PCR or/and Western blots were performed to examine the expression of CD44, $\beta$-catenin, Sox-2, PDCD4, CK-20 and k-Ras. Colonosphere formation and SCID mice xenograft studies were performed to evaluate the tumorigenic properties of CSC-enriched colon CR cells.

Results: We investigated the role that microRNAs (miRs), specifically miR-21 and miR-145 play in regulating colon CSCs. We found the expression of miR-21 to be greatly increased and miR-145 decreased in CR colon cancer cells that are highly enriched in CSC, indicating a role for these miRNAs in regulating CSCs. In support of this, we found that whereas forced expression of miR-145 in colon cancer cells greatly inhibits CSCs and tumor growth, up-regulation of miR-21 causes an opposite phenomenon. In addition, administration of mature miR-145 or antagomir-21 (anti-sense miR-21) greatly suppresses the growth of colon cancer cell xenografts in SCID mice. This was associated with decreased expression of CD44, $\beta$-catenin, Sox-2 and induction of CK-20 indicating that administration of miR-145 or antagomir-21 decreases CSC proliferation and induces differentiation. In vitro studies further demonstrate that miR-21 negatively regulates miR-145 and vice versa. $k$-Ras appears to play critical role in regulation of this process, as evidenced by the fact that the absence of $\mathrm{k}$-Ras in CR colon cancer cells increases miR-145 expression, suppresses miR-21, and interrupts the negative cooperation between miR-21 and miR-145.

Conclusions: Our current observations suggest that miR-21, miR-145, and their networks play critical roles in regulating CSCs growth and/or differentiation in the colon cancer and progression of chemo-resistance.

Keywords: Colorectal cancer, Negative feedback, Chemo-resistance

\section{Background}

Despite advances in medicine, nearly $50 \%$ of patients with colorectal cancer (CRC) show tumor recurrence, for which the outcome remains poor; the median survival following recurrence is only 13.3 months [1]. The higher recurrence of CRC is thought to be the result of drug-resistance of cancer cells. Although the reasons for drug resistance are

\footnotetext{
*Correspondence: aa5142@wayne.edu; a.majumdar@wayne.edu

'Department of Veterans Affairs Medical Center, 4646 John R, Detroit, Ml 48201, USA

Full list of author information is available at the end of the article
}

not fully understood, they may be related to the presence of cancer stem or cancer stem-like cells (CSCs/CSLCs) that are thought to play pivotal role in tumor initiation, progression, metastasis, and its relapse [2-4].

CSCs/CSLCs are a small population of self-renewing undifferentiated cells within a tumor that have been shown to be resistant to radiation and chemotherapy [2]. CSCs/ CSLCs isolated from different solid tumors, including the colon are usually identified by specific surface epitopes. Colon CSCs/CSLCs have been shown to express CD44, CD166, CD133 and ESA (epithelial-specific antigen, also 
known as EpCAM) surface markers [5]. We have observed that in humans, colon CSCs/CSLCs are present not only in premalignant adenomas but also in normal appearing colonic mucosa and that the population of CSCs/CSLCs increases with advancing age, suggesting that they may be partly responsible for the age-related rise in colorectal cancer [6].

The standard therapy for advanced CRC includes surgery followed by chemotherapy or other effective therapeutic regimen to eliminate any remaining cancer cells. 5Fluorouracil (5-FU) based regimen such as FOLFOX (5-FU plus Oxaliplatin and Folinic acid) remains the backbone of colorectal cancer chemotherapeutics but with limited success. Near 50\% tumor recurrence rate suggests that the cells survive chemotherapy and may lead to cancer recurrence. We have reported that 5-Fluorouracil and Oxaliplatin (FU$\mathrm{Ox}$ ) resistant [chemo-resistant (CR)] colon cancer HCT116 and HT29 cells exhibit enrichment of CSCs/CSLCs, elevated levels of mature miR-21 and that miR-21 induces stemness in colon cancer cells $[7,8]$.

MicroRNAs (miRNAs) comprise a broad class of small (19-22 nucleotide) endogenous RNAs that negatively control the expression of the target genes by cleaving mRNA or through translation repression [9], and can function as oncogenes or tumor suppressors depending on the target. More than 1500 human miRNAs are annotated in the miRBase and many of them are aberrantly expressed in several pathological conditions, including cancer. In colorectal cancer, miR-21 has been reported to function as an oncomiR (a miRNA with oncogenic properties) due to its key role in several processes of tumor promotion, invasion and metastasis $[10,11]$. We found miR-21 to induce stemness of colon cancer cells [8]. Furthermore, overexpression of miR-21 has been shown to dramatically reduce the therapeutic efficacy of 5-FU [12]. Although the underlying mechanisms for regulation of miR-21 in CRC remain to be defined, we reported EGFR inhibitor Cetuximab (mAb to EGFR) to decrease its expression suggesting a role for EGFR in regulating miR-21[13]. In contrast to miR-21, miR-145 is a p53 regulated tumor suppressor, whose down-regulation has been found in colorectal and other cancers $[14,15]$. It regulates stem cell renewal and pluripotency by suppressing multiple pluripotent genes: OCT4, SOX2 and KLF4 [14].

Herein, we report that stable over expression of miR145 in colon cancer HCT-116 or HT-29 cells or in the corresponding CR colon cancer cells significantly induces differentiation and inhibits their growth in vitro. A similar phenomenon occurs following down-regulation of miR-21 in CR colon cancer cells. The tumorigenic potential of parental and CR-HT-29 cells in SCID mice was inhibited by administration of miR-145 or anti-miR-21. In addition, we observed downregulation of pluripotency factors Oct4, Sox2, Nanog as well as miR-21 following overexpression of miR-145 in colon cancer cells. We also report that miR21 negatively regulates miR-145 and vice versa. k-Ras appears to play critical role in the regulation of this process, as evidenced by the fact that the absence of k-Ras in CR colon cancer cells increases miR-145 expression, suppresses miR-21, and interrupts the cooperation between miR-21 and miR-145.

\section{Results}

Over-expression of miR-145 induces differentiation, inhibits stemness and xenograft tumors in SCID mice

More than $80 \%$ of colorectal cancers arise from adenomatous polyps that are known to contain CSCs/CSLCs [6] and dysregulation of miRNAs [16]. We have reported that the expression of miR-21 is greatly increased in chemo-resistant (CR) colon cancer cells that are highly enriched in CSC, and forced expression of miR-21 in colon cancer cells greatly increases CSC population accompanied by induction of tumor growth, indicating miR-21 regulates stemness of colon cancer cells $[8,17]$.

To determine the putative functional properties of miR145 in the development of colorectal tumor and its relation to miR21 expression, $\mathrm{pCMV} / \mathrm{miR} 145$ plasmid (Origene, Rockville, $\mathrm{MD}$ ) was stably transfected in HCT-116 cells. As determined by qRT-PCR (real time PCR) analysis, the expression of miR-145 was found to be 4-fold higher in the miR-145 positive cells, compared to empty vector (Figure 1A). In contrast, miR-21 was decreased by $50 \%$ in miR-145 overexpressing cells, compared to the vector-transfected control cells (Figure 1A). Western blot analysis revealed that the levels of Sox 2 , the target of miR-145 were decreased by $34 \%$ and the expression of cytokeratin-20 (CK-20), the differentiation marker was increased by $67 \%$, compared to the vector-transfected controls (Figure 1B).

The next set of experiments was carried out to determine whether miR-145 may regulate stemness of colon cancer cells. The functional property of cancer stem cells is defined by their ability to form sphere/spheroid (in vitro) in serumfree medium containing growth factors (stem cell medium) when plated in ultra low-attachment plates under extreme limited dilutions [18]. To determine whether and to what extent miR-145 over-expression affects the sphere forming properties of colon cancer cells, HCT-116 cells stably transfected with $\mathrm{pCMV} / \mathrm{miR}-145$ or $\mathrm{pCMV}$ vector (control) were subjected to an extreme limiting dilution analysis (ELDA). Number of spheroids formed as well as the frequency to form spheroids by miR-145 expressing HCT-116 cells was found to be about $88 \%$ lower than those formed by the vector-transfected control cells $(\mathrm{p}<0.001)$ (Figure $1 \mathrm{C}$ and Table 1). Further, the average diameter of spheroids, obtained from miR-145 overexpressing cells, was found to be smaller than the controls (Table 1 and Figure 1C). Tumorigenic potential of the miR-145 overexpressing cells was analyzed by generating xenograft tumors in SCID mice. 

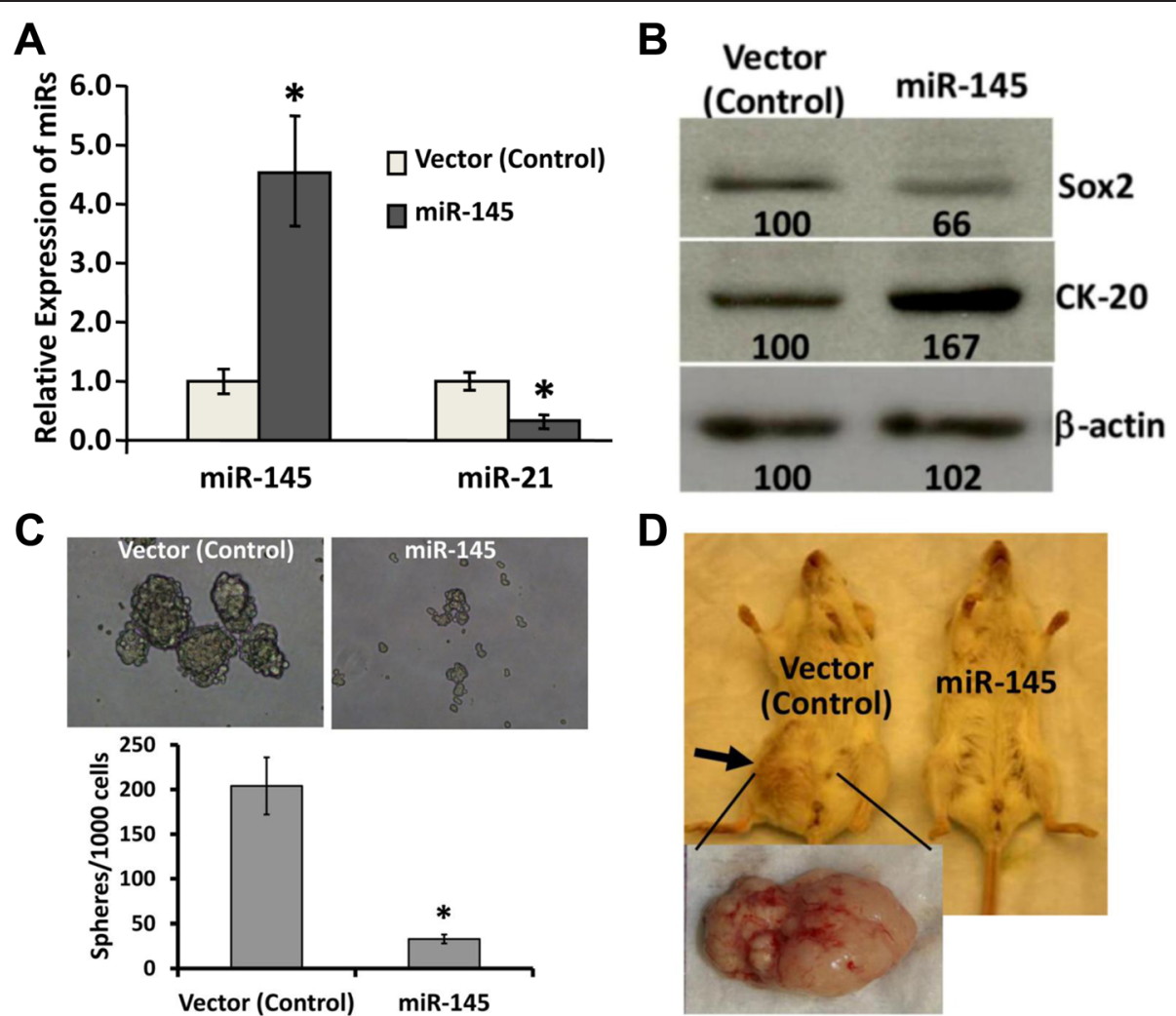

Figure 1 Over-expression of miR-145 in colon cancer HCT-116 cells by stably transfected pCMV/miR-145 downregulates miR-21 and induces differentiation, inhibits stemness and produces no visible tumors in SCID mice. (A) qRT-PCR showing up-regulation of mature miR-145 and down regulation of miR-21 in HCT-116 cells that were stably transfected with pCMV/miR-145 plasmid or the corresponding vector, ${ }^{*} P<0.001$. (B) Western-blot showing decreased expression of Sox2 and increased expression of CK-20 in miR-145 over-expressing HCT-116 cells, compared to the corresponding vector-transfected control cells. $\beta$-actin was used as a loading control, (C) Representative photographs showing colonospheres formed by the cells from miR-145 expressing clones, derived from HCT-116 cells, stably transfected with pCMV-miR-145 plasmid or the corresponding vector (top panel), histogram showing the number of colonspheres formed by miR-145 over-expressing HCT-116 cells, compared to the corresponding vector-transfected control cells (bottom panel, ${ }^{*} P<0.001$ ), (D) while administration of miR-145 over-expressing HCT-116 cells produces no visible tumors in SCID mice, the vector-transfected HCT-116 cells (control) induces large tumors in SCID mice. The data represent means of three independent experiments.

We observed that while the HCT-116 pCMV empty vector-transfected (control) cells formed palpable tumor within 3 weeks of injection, no tumor could be detected with HCT-116 pCMV/miR-145 cells even after 6 weeks (Figure 1D).

miR-145 and miR-21 cooperation plays a role in regulating cancer stem cell proliferation and differentiation

In the next set of experiments we tested whether there is a cooperation between miR-145 and miR-21 and how this cooperation may regulate cancer stem cell proliferation and differentiation. To conduct this experiment, we utilized CR colon cancer cells that are enriched in CSCs and exhibit a $3-5$ fold increase in the precursor and mature miR-21 as shown in Figure 2A, an observation similar to what we reported earlier $[8,17]$. We observed that the expression of tumor suppressive miR-145 in CR HCT-116 cells was decreased by $62 \%$ and in miR-21-overexpressing HCT-116 cells by $90 \%$, when compared with their corresponding parental or empty vector control cells (Figure $2 \mathrm{~A}$ and B). In

Table 1 Sphere-forming frequency, diameter and/or self-renewal ability of cells from colonospheres of stably transfected with pCMV (Vector), pCMV/miR-145 (miR-145) colon cancer HCT-116 cells

\begin{tabular}{llll}
\hline & Vector & miR-145 & $P$ value \\
\hline Sphere-forming frequency $(95 \% \mathrm{Cl})$ & $1 / 11(1 / 17-1 / 4)$ & $1 / 88(1 / 196-1 / 40)$ & $<0.001$ \\
Diameter $(\mu \mathrm{m})$ & $104.0 \pm 18.5$ & $47.0 \pm 13.2$ & $<0.001$ \\
Self-renewal (spheres per 200 cells) & $13 \pm 3$ & - & $<0.001$
\end{tabular}

Data are pooled from three independent experiments for each. $\mathrm{Cl}$, confidence interval. 

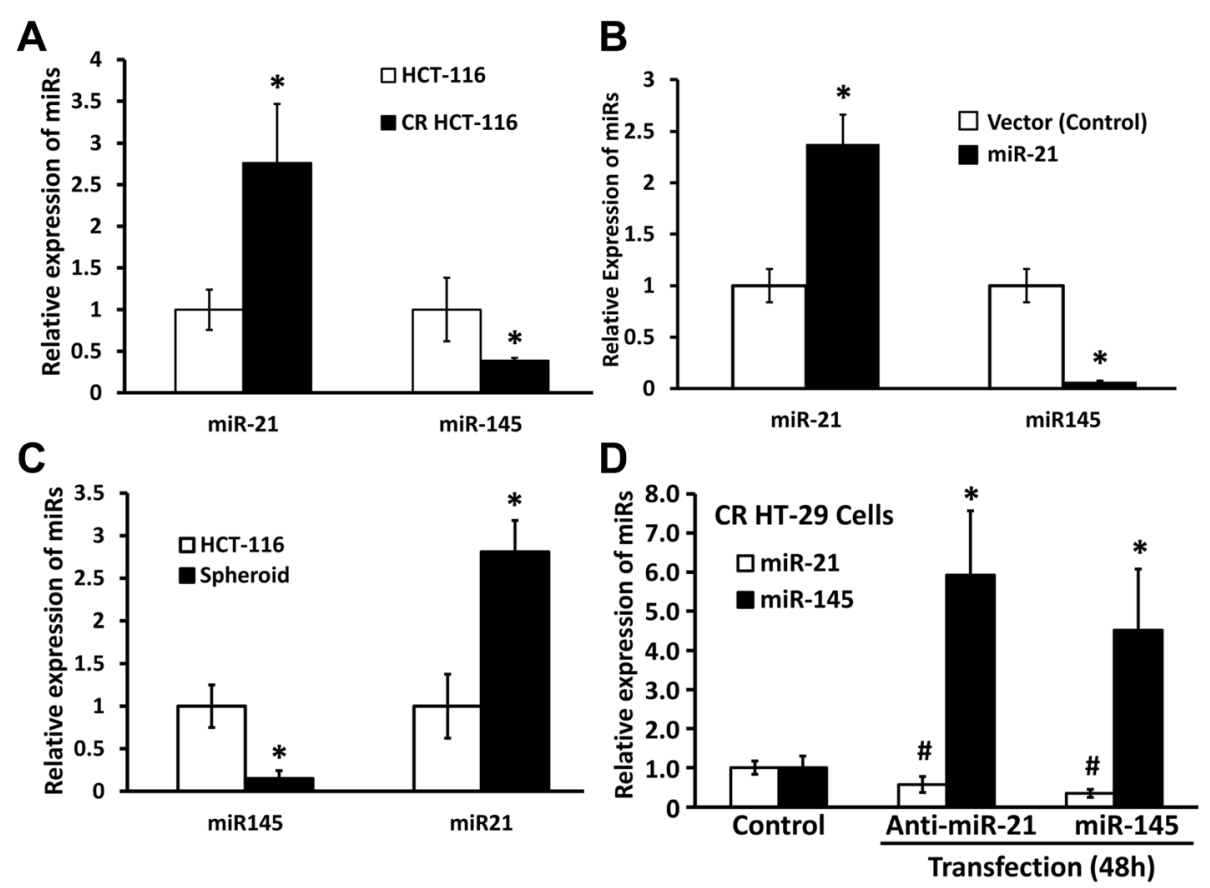

Figure 2 Chemo-resistance, overexpression of miR-21 and colonosphere formation in colon cancer HCT-116 cells, all of which are associated with increased miR-21 levels, lead to down-regulation of miR-145, and miR-145 and miR-21 negatively regulate each other in chemo-resistant (CR) colon cancer cells. (A) qRT-PCR showing up-regulation of mature miR-21 and down-regulation of mature miR-145 in CR HCT-116 cells when compared with the parental HCT-116 cells. (B) qRT-PCR showing over-expression of miR-21 (stably transfected cells) leads to decreased miR-145 compared to the vector-transfected control. (C) qRT-PCR showing down-regulation of miR-145 and up-regulation of mature miR-21 in colonospheres formed by parental HCT-116 cells. (D) qRT-PCR showing relative expression of miR-21 and miR-145 in CR HT-29 cells at $48 \mathrm{~h}$ following transfection of mature miR-145 or anti-miR-21. All the data represent means \pm SD of three independent experiments, ${ }^{*} P<0.001$, compared to the control.

addition, we observed that while the levels of miR-145 were decreased by $\sim 70 \%$ in colonospheres formed by the parental HCT-116 cells, the expression of miR-21 was increased by $\sim 180 \%$ in these colonospheres, when compared with the corresponding parental HCT-116 cells (Figure 2C). These results indicate that overespression of miR-21 is associated with downregulation of miR-145 and vice versa in colon cancer cells and that both miR-21 and miR-145 are involved in regulating the growth of colonospheres, enriched in CSCs.

To further investigate the cooperation between miR-145 and miR-21 in CR colon cancer cells, we regulated the levels of miR-145 (increased) or miR-21(decreased) by transfecting mature miR-145 or anti-miR-21 in CR-HT-29 cells in vitro. As expected, miR-145 was increased and miR-21 was decreased after transfection of miR-145 and anti-miR-21, compared with corresponding control in CR colon cancer cells (Figure 2D). Following transfection of anti-miR21 in CR-HT-29 cells, there was a marked 6-fold increase of miR-145 (Figure 2D). On the other hand, in miR145-transfected CR HT-29 cells, the levels of miR21 were decreased by $70 \%$, compared to the corresponding control (Figure 2D). Collectively, the current data suggest a negative feedback between the tumor suppressor miR145 and oncomiR miR-21 in CR colon cancer cells.
miR-145 and, anti-miR-21 inhibit tumorigenic potential of chemo-resistant (CR) colon cancer HT-29 cells in SCID mice

To determine whether and to what extent miR-145 or antimiR-21 would affect the tumorigenic potential, xenografts in SCID mice formed by colon cancer parental HT-29 or CRHT-29 cells were first analyzed for the presence of markers of CSCs/CSLCs and their growth and differentiation. We observed that the expression of CD44 (colon CSC marker), $\beta$-catenin (stem cell growth regulator) and SOX2 (a miR145 target) was 39\%, 356\% and 1600\% higher, respectively, whereas the levels of PDCD4 and CK20 were 40\% and 95\% lower in CR HT-29 xenografts, when compared with the values from xenografts of parental HT-29 cells (Figure 3).

We then determined the effectiveness of miR-145 in suppressing the growth of colon cancer xenografts in SCID mice. HCT-116 cells have consistently active mutated k-Ras, while HT-29 cells harbor normal k-Ras. Additionally, endogenous miR-145 is highly repressed in HT-29 cells, therefore, in this investigation, $2.5 \times 10^{5} \mathrm{CR}$ HT-29 cells were injected with 50\% Matrigel in each mouse. Once palpable tumors were developed ( $\sim 3$ weeks), Polyethylenimines (PEI)/miRNA-145 complex treatment (i.p.; weekly) was initiated. The results revealed that treatment with (PEI)/miRNA-145 complex significantly 


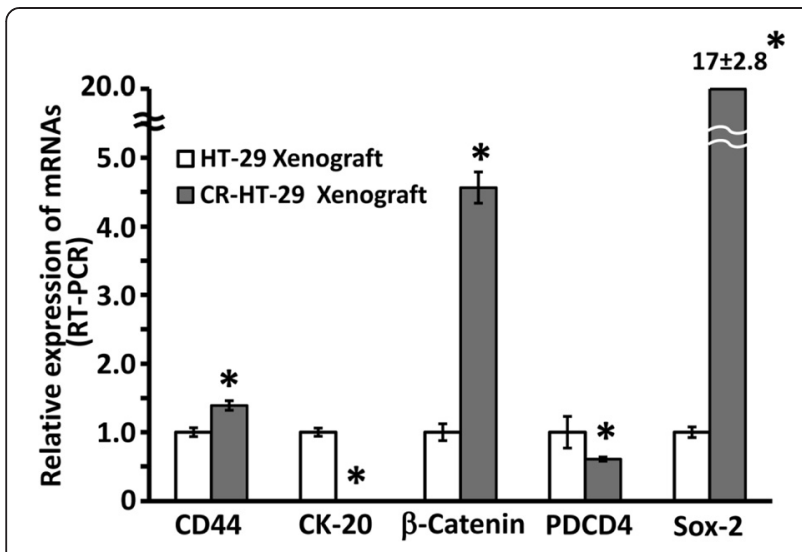

Figure 3 Presence of markers of CSC and their growth and differentiation in xenografts derived from parental HT-29 or CR HT-29 cells. qRT-PCR showing relative expression of CD44, CK20, $\beta$-Catenin, PDCD4 and Sox2 in xenografts derived from parental HT-29 or CR HT-29 cells. The data represent means \pm SD of three independent experiments, ${ }^{*} P<0.001$, compared to the control.

suppressed tumor growth, compared with the vehicletreated controls (Figure 4A). The relative level of miR-145 in the tumor of SCID mice injected with miR-145 was about 50\% higher than the control tumor (Figure 4A; lower panel).

At the end of the experiment, a small portion of the tumors from CR-HT-29 cells and those treated with (PEI)/miRNA-145 or vehicle complex was used for RNA extraction and analyzed for the expression of various markers of CSC growth and differentiation by real time PCR. The expression of CD44, $\beta$-catenin and SOX 2 was decreased by $45 \%, 34 \%$ and $36 \%$, and PDCD 4 and CK20 was increased significantly by $10 \%$ and over $4000 \%$, respectively, in CR-HT-29 xenografts from (PEI)/miRNA145 complex-treated xenograft, compared to those fromed by vehicle- treated (control) CR HT-29 cells (Figure 4B). These results indicate that administration of miR-145 greatly decreases CSC proliferation and induces differentiation leading to suppression of tumor growth in SCID mice.

\section{Discussion}

Although many factors may contribute to colorectal cancer, chemo-resistance and relapse of cancer and metastasis, it is reasonable to speculate that CSCs play critical role in these processes. Our current data further suggest that the tumor suppressor miR-145, oncomiR miR-21 and their networks are critically involved in regulating these events.

Earlier, we reported that exposure of colon cancer HCT116 or HT-29 cells to the combination of 5-Fluorouracil (5-FU) and Oxaliplatin (Ox), the backbone of colorectal cancer chemotherapy, inhibited their growth and led to enrichment of CSC/CSLC phenotype, where the levels of miR-21 were greatly increased indicating a potential role for

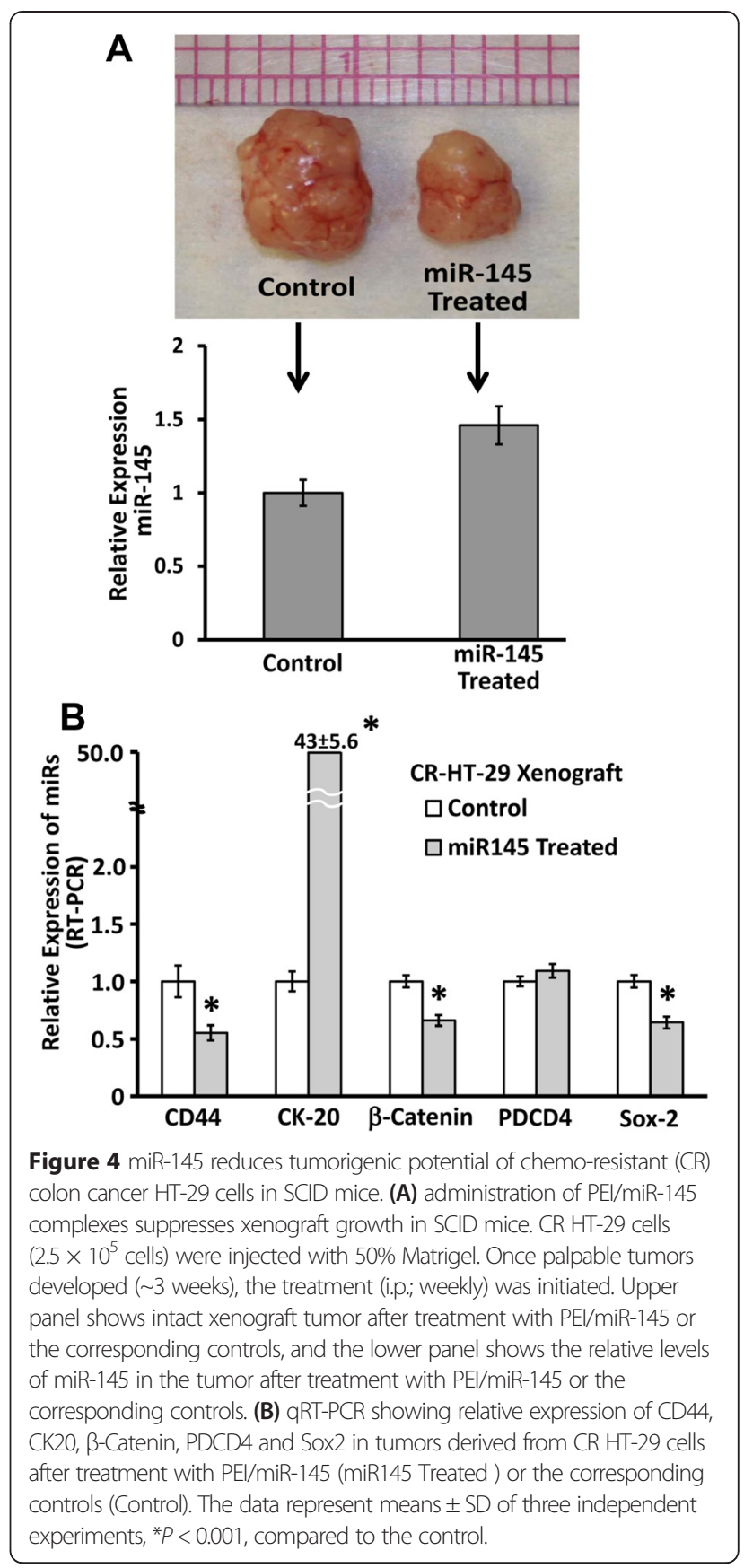

this miR in regulating CSCs/CSLCs $[7,8]$. In addition, we reported miR-21 to induce stemness of colon cancer cells by down-regulating TGF $\beta R 2$ and that down-regulation of miR21 induces differentiation of CR colon cancer cells and enhances susceptibility to therapeutic regimens $[8,17]$.

We also demonstrated that miR-21 regulates CSC function as well as its growth and differentiation by modulating its direct targets such as TGF $3 R 2$, PDCD4 and PTEN, as reported earlier $[8,17,19]$. Our current data show that miR-21 also regulates the expression of the tumor suppressor miR145. The latter is supported by the following observations: 
(a) miR-21 is up-regulated, whereas miR-145 is downregulated in colon cancer CR-HCT-116 cells, highly enriched in CSCs/CSLCs; (b) forced expression of miR-21 through transfection of mature miR-21 in colon cancer HCT-116 cells decreases the expression of miR-145; (c) knock-down of miR-21 by anti-miR-21 increases miR-145 in CR colon cancer cells. These results indicate that miR-21 negatively regulates the expression of miR-145 in colon cancer cells. On the other hand, miR-21 can be decreased by elevating the levels of miR-145 through transfection of mature miR-145 in parental and CR colon cancer cells. These data further suggest that a negative feedback exists between miR-21 and miR-145.

miR-145 is a p53-regulated gene. p53 can induce its transcription and enhance the post-transcriptional maturation of miR-143/miR-145 cluster $[15,20]$ in response to DNA damage by interacting with the Drosha processing complex [21]. Activated Ras can suppress miR-143/145 cluster transcription through Ras-responsive element-binding protein (RREB1), which represses the miR-143/145 promoter [22]. Hatley and colleagues have reported that miR-21 increases RAS signaling activity and therefore leads to repression of the miR-143/145 cluster [23]. These reports support our data and further demonstrate the mechanism by which miR-21 negatively regulates tumor suppressor miR-145.

Down-regulation of miR-145 has been found in multiple tumors including colon, breast, prostate, pancreas etc. $[14,15]$. In fact, miR-145 has been well documented as a tumor suppressor gene because it negatively regulates multiple oncogenes such as Myc, K-Ras, IRS-1, ERK5 [15,24]. Moreover, miR-145 negatively regulates junctional cell adhesion molecule (JAM-A), fascin and MUC1 and suppresses breast cancer cell motility and invasiveness [25,26]. miR-145 also inhibits colon cancer cells' proliferation and sensitizes them to 5-fluorouracil by targeting oncogenic FLI1 [27].

miR-21 expression is regulated at multiple levels, including transcription and post-transcriptional processing. Talotta et al. [28] have reported that the miR-21 is induced by AP1 in response to Ras. They have also demonstrated that PDCD4, a pro-apoptotic gene and the target of miR-21, is a negative regulator of AP1. The miR21-mediated down-regulation of PDCD4 is essential for the maximal induction of AP1 activity in response to Ras. These data reveal a mechanism of positive autoregulation of the AP1 complex in Ras transformation and disclose the function of oncomiRs as critical targets and regulators of AP1 in tumorigenesis [29]. Kern et al. [30] also showed that EGF/Ras efficiently induced the miR-21 primary transcript, but this does not rapidly and simply translate into higher mature miR-21 levels.

In view of these reports together with our current observations prompted us to hypothesize that the negative feedback between miR-21 and miR-145 mediated by Ras signaling pathway plays a crucial role in the induction of
CSC proliferation or/and differentiation in CR colon cancer cells, as depicted in Figure 5A. Restoration of miRs level by increasing miR-145 or decreasing miR-21 can dislodge Ras mediated feedback between miR-145 and miR-21 and inhibit tumor growth. Indeed, this hypothesis has been supported by our in vitro (Figure 5B-D) and in vivo experiments (Figure 4). In the in vitro studies, downregulation of k-Ras in CR colon cancer cells was achieved by transfection with corresponding siRNA and the controls with scrambled siRNA. The protein as well as mRNA levels of $\mathrm{k}$-Ras were substantially reduced (70-80\% reduction), as determined by Western blot and qRT-PCR analyses, when compared with the corresponding controls (Figure $5 B$ ). Down-regulation of k-Ras in CR HT- 29 cells, that caused a $45 \%$ increase in miR145 , produced a $35 \%$ reduction in miR-21, when compared with the corresponding controls (Figure 5C). Forced expression of miR-21 in k-Ras downregulated CR-HT-29 cells resulted in 1-fold increase in miR-145 (Figure 5D). But, increasing the levels of miR-145 in k-ras downregulated CRHT-29 cells resulted in no significant reduction in miR-21, compared with corresponding control (Figure 5D). These observations are in contrast to those depicted in Figure 2D, where are show that in cell with intact k-Ras, downregulation of miR-21 produced 6-fold augmentation of miR-145, forced expression of miR-145 resulted in 70\% reduction in miR-21. These observations suggest that $\mathrm{k}$-Ras mediates the negative feedback between the tumor suppressor miR-145 and oncomiR miR-21 in CR colon cancer cells. This inference is further supported by our in vivo experiments which demonstrate that increase of miR-145 or inhibition of miR21 by injecting PEI mediated chemically engineered modified single-stranded RNA or analogues complementary to miRNA which are efficient, specific and long-lasting replacements or silencers of endogenous miRNAs in mice [24,31] leads to suppression of xenograft growth in SCID mice. The growth inhibition was associated with decreased proliferation of CSCs and induction of their differentiation, as evidenced by reduction in expression of CD44 and induction of CK20 following upregulation of miR-145 or downregulation of miR-21.

\section{Conclusions}

Our current observations suggest that dysregulation of miR-21 and miR-145 plays a central role in the growth of CSCs in chemo-resistant colon tumors by regulating a network of genes that are critically involved in tumor progression, metastasis, and relapse of colorectal cancer.

\section{Materials and methods \\ Cell lines and cell cultures}

Human colon cancer HCT-116 and HT-29 cells were obtained from the American Type Culture Collection (ATCC, Rockville, MD). They were expanded and frozen in aliquots. 


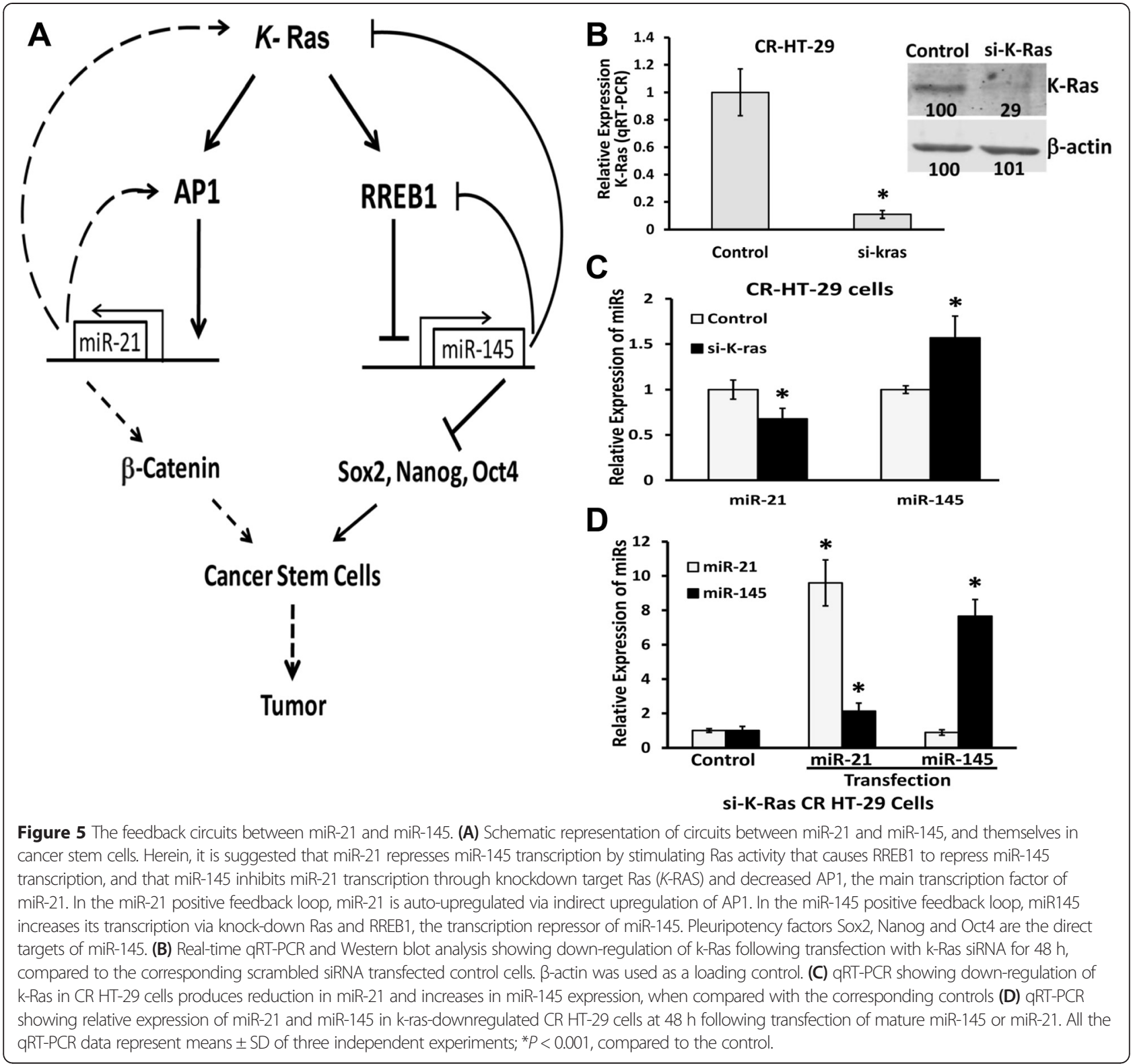

Fresh aliquots were used every 6-7 months. The cells were maintained in Dulbecco's modified Eagle medium (DMEM; $4.5 \mathrm{~g} / \mathrm{L}$ d-glucose) supplemented with 10\% FBS (Invitrogen, Grand Island, NY) and 1X antibiotic/antimycotic (Invitrogen, Grand Island, NY) in tissue culture flasks, 5-Fluorouracil and Oxaliplatin (FuOx) resistant [chemo-resistant (CR)] colon cancer HCT116 and HT29 cells were generated as described earlier $[7,8]$ in our laboratory and were maintained in normal culture medium containing $2 \times \mathrm{FuOx}(50 \mu \mathrm{M} 5-\mathrm{Fu}+1.25 \mu \mathrm{M} \mathrm{Ox})$ in tissue culture flasks in a humidified incubator at $37^{\circ} \mathrm{C}$ in an atmosphere of $95 \%$ air and $5 \%$ carbon dioxide. The medium was changed two times a week, and cells were passaged using 0.05\% trypsin/EDTA (Invitrogen, Grand Island, NY).

\section{Western blot analysis}

Western blot analysis was performed according to our standard protocol [32,33]. Briefly, the cells were solubilized in lysis buffer and the protein concentration was determined by the Bio-Rad Protein Assay kit (Bio-Rad, Hercules, CA). The proteins were separated by SDSPAGE and transferred to PVDF membranes (Millipore). The membranes were incubated overnight at $4^{\circ} \mathrm{C}$ with primary antibodies after blocking. The membranes were subsequently washed and incubated with appropriate secondary antibodies. The protein bands were visualized by enhanced chemiluminescence (ECL) detection system (Amersham, Piscataway, NJ). Whenever appropriate, the membranes were stripped and re-probed with $\beta$-actin for verification of protein loading. 
Transfection of miR-145, miR-21, anti-miR-21 or k-Ras siRNA in colon cancer cells

For transfection of miR-21/145, anti-miR-21 or/and siRNA in the CR-HCT-116 or CR-HT-29 cells, Lipofectamine 2000 transfection reagent (Invitrogen Corp.) and serum-free Opti-MEM (Invitrogen Corp.) medium were prepared according to the manufacturer's instructions. Briefly, the cells were plated in six-well tissue culture plates with normal growth medium and incubated overnight to achieve 40-60\% confluence. Next day, the medium was removed, washed with serum-free OptiMEM (Invitrogen Corp.) medium prior to adding the complexes containing 100 pmol of scrambled (control), kRas siRNA (Integrated DNA Technologies, Coralville, IA) or/and pre-miR-145, pre-miR-21. After 2 days of transfection, the cells were collected and analyzed for protein expression of k-Ras using western blot and for quantitation of miRNA-21 and miR-145 by qRT-PCR according to our standard protocol.

\section{Isolation of RNA and quantitative polymerase chain reaction analysis}

Total RNA was extracted from different cells using RNA-STAT solution (Tel Test, Friendswood, TX) according to the manufacturer's instructions. The total RNA was treated with DNase I and purified with phenol-chloroform. RNA concentration was measured using a NanoDrop 2000C spectrophotometer.

Quantitative reverse transcription-polymerase chain reaction (qRT-PCR) was performed using the GeneAmp RNA PCR Kit (Applied Biosystems, Foster City, CA). $5 \mu \mathrm{l}$ of cDNA products were amplified with SYBR Green Quantitative PCR Master Mix (Applied Biosystems). PCR primers used were as follows: CD44, forward: $5^{\prime}$-aaggtggag caaacacaacc-3', reverse: 5' -actgcaatgcaaactgcaag-3'; CK-20, forward: $5^{\prime}$-tgaagagctgcgaagtcaga- $3^{\prime}$ and reverse: $5^{\prime}$-gaagtc ctcagcagccagtt-3', $\beta$-Catenin forward: 5 '-ggaaggtctccttggga ctc-3' and reverse: 5'-ataccacccacttggcagac-3'; PDCD4, forward: 5'-ggtgggccagtttattgcta-3' and reverse: 5'-cggtacccttatccagagca-3'; Sox2, forward: 5'-aaccccaagatgcacaactc-3' and reverse: 5'-gcttagcctcgtcgatgaac-3'; k-Ras, forward: 5'-tgtgg tagttggagctggtg-3' and reverse: 5'-tccaagagacaggtttctcca-3'; $\beta$ actin forward: $5^{\prime}$-cccagcacaatgaagatcaa- 3 ' and reverse $5^{\prime}$-ac atctgctggaaggtggac $-3^{\prime}$. Reactions were carried out in Applied Biosystems 7500 Real-Time PCR System; The running conditions for PCR were as follows: for activating the DNA polymerase, hot start was performed for $10 \mathrm{~min}$ at $95^{\circ} \mathrm{C}$, and then cycling at $95^{\circ} \mathrm{C}$ for $15 \mathrm{~s}$ and $60^{\circ} \mathrm{C}$ for $1 \mathrm{~min}$ for a total of 40 cycles.

\section{Quantitation of miRNA-21 and miR-145}

TaqMan microRNA assays were used to quantitate miR21 and miR-145 in different colon cancer cells according to the manufacturer's instructions (Applied Biosystems,
Foster City, CA). Briefly, cDNA synthesis was carried out with the TaqMan MicroRNA reverse transcription kit (Applied Biosystems). The miRNA reverse transcriptionPCR (RT-PCR) primers for $m i R-21, m i R-145$ and endogenous control RNU6B were purchased from Applied Biosystems. Real-time quantitative RT-PCR (qRT-PCR) analysis was carried out using Applied Biosystems 7500 Real-time PCR System. The PCR mix containing TaqMan $2 \times$ Universal PCR Master Mix were processed as follows: $95^{\circ} \mathrm{C}$ for $10 \mathrm{~min}$ and then $95^{\circ} \mathrm{C}$ for $15 \mathrm{~s}, 60^{\circ} \mathrm{C}$ for $60 \mathrm{~s}$ for up to 40 cycles. Signal was collected at the endpoint of every cycle. The gene expression $\Delta C_{\mathrm{T}}$ values of miRNAs from each sample were calculated by normalizing with internal control RNU6B and relative quantitation values were plotted.

\section{Generation of miR-145 over-expressing HCT-116 cells}

pCMV-miR-145 plasmid carrying pre-microRNA-145 and 250-300 nts up and down-stream flanking sequence (Origene, Nockville, MD) or empty vector DNA alone (pCMV) was transfected into HCT-116 cells by Lipofectamine ${ }^{\mathrm{TM}}$ 2000 reagent according to the manufacturer's instructions (Invitrogen Corp, CA). Several independent sublines (colonies) were generated over $8-10 \mathrm{wk}$ of the selection period in the presence of $0.6 \mathrm{mg} / \mathrm{ml} \mathrm{G418} \mathrm{(Neomycin).} \mathrm{Colonies}$ were picked at random and grown as individual cell lines in the presence of $0.4 \mathrm{mg} / \mathrm{ml} \mathrm{G418}$. Each cell line was subjected to RT-PCR analysis to evaluate miR-145 expression.

\section{Formation of colonospheres and Extreme limiting dilution analysis}

The ability of miR-145-overexpressing and parental HCT116 cells to form spheres in suspension was evaluated as described previously [18]. Briefly, primary colonospheres were generated by incubating the limited number of pCMV and miR-145 stably transfected HCT-116 cells at a concentration of 1000 cells per $500 \mu \mathrm{L}$ in serum-free stem cell medium (SCM) containing DMEM/F12 (1:1) supplemented with B27 (Life Technologies, Gaithersburg, MD), $20 \mathrm{ng} / \mathrm{ml}$ EGF (Sigma, St Louis, MO), $10 \mathrm{ng} / \mathrm{ml}$ fibroblast growth factor (Sigma), and antibiotic-anti-mycotic in 24well plates (Corning Inc, Lowell, MA) for 10 days. The formed colonospheres were centrifuged (1000 rpm), dissociated with $0.05 \%$ trypsin/EDTA and reseeded in SCM. The single-cell suspension derived from colonospheres that have undergone 15 or more serial passages were used for all experiments.

Self-renewing/regeneration abilities of the spheres, derived from pCMV and miR-145 stably transfected HCT-116 cells, were analyzed for secondary colonospheres formation in the following manner. Primary colonospheres formed over a period of ten days in SCM containing DMEM/F12 (1:1) were collected by centrifugation, dissociated with $0.05 \%$ trypsin/EDTA, and subsequently passed through a $40 \mu \mathrm{M}$ 
sieve to obtain single cell suspensions. An equal number of cells from primary colonospheres culture were plated (100 cells/500 $\mu \mathrm{L}$ in SCM) in ultra low-attachment wells. The secondary colonospheres formed after five days were recorded for their number and size by light microscopy.

Extreme limiting dilution analysis (ELDA) was performed essentially according to $\mathrm{Hu}$ and Smyth with slight modifications as described by us [18,34]. Briefly, single cell suspension obtained from adherent miR-145-expressing HCT-116 and vector-transfected (control) HCT-116 cells were plated at concentrations of 200, 20 and 2 cells per $100 \mu \mathrm{l} \mathrm{SCM} \mathrm{(24} \mathrm{well} \mathrm{for} \mathrm{each} \mathrm{dilution)} \mathrm{in} \mathrm{96-well} \mathrm{plates}$ and incubated for 5 days. At the end of 5 days, the number of wells showing formation of colonospheres was counted. The frequency of sphere formation in a particular cell type was determined using ELDA webtool at http://bioinf.wehi.edu.au/software/elda.

\section{SCID mice xenografts}

The research protocol was approved by Wayne State University and the VA Medical Center Institutional Animal Care and Use Committees. Four-week-old female ICR/severe combined immunodeficient mice (SCID), obtained from Taconic Laboratory (Germantown, NY) were used for these studies.

To determine the tumorigenic potential of miR-145 overexpressing HCT-116 cells, SCID mice were subcutaneously (s.c.) injected with $\sim 2.5 \times 10^{5}$ HCT-116 cells that were stably transfected with pCMV/miR-145 or pCMV (control).

To further determine whether and to what extent forced expression of miR145 or down-regulation of miR-21 by anti-miR21 would affect the tumorigenic potential of colon cancer cells, SCID mice were subcutaneously injected either with $\sim 2.5 \times 10^{5}$ CR HCT-116 or CR HT29 cells suspended in $100 \mu \mathrm{l}$ Matrigel. Once palpable tumors were formed, SCID mice bearing xenografts were treated with PEI/miRNA-145, PEI/anti-miRNA-21 complexes or $\mathrm{PEI} /$ control once a week by i.p. injection of $0.45 \mathrm{nmol}(6 \mu \mathrm{g})$ for 3 weeks [24]. Tumor measurements were carried out at multiple time points during the experimental period. The experiment was repeated 3 times with 3-5 mice in each group. Mice were weighed regularly to monitor their well being, and the tumor volumes were estimated as follows: volume $\left(\mathrm{mm}^{3}\right)=\left(L \times \mathrm{W}^{2}\right) / 2$, where $\mathrm{L}$ and $\mathrm{W}$ are the tumor length and width (in $\mathrm{mm}$ ), respectively.

\section{Statistical analysis}

Unless otherwise stated, data are expressed as mean \pm SEM. Wherever applicable, the results were analyzed using analysis of variance followed by Fisher protected least significant differences or Scheffé test. $p<0.05$ was designated as the level of significance.

\section{Abbreviations}

CRC: colorectal cancer; CSCs/CSLCs: cancer stem or stem-like cells; 5-FU: 5-Fluorouracil; Ox: Oxaliplatin; FU-Ox: 5-FU and Oxaliplatin; CR: chemo-resistant; CK-20: cytokeratin-20; RREB1: Ras-responsive element-binding protein 1; ELDA: Extreme limiting dilution analysis; qRT-PCR: quantitative reverse transcription-polymerase chain reaction; PEl: Polyethylenimines.

\section{Competing interests}

The authors declare that they have no competing interests.

\section{Authors' contributions}

YY carried out majority of the experiments, wrote the first draft of the manuscript and responsible for planning, designing, analysis of the data. He was helped by PNM, SG and LF. PNM and EL was involved in the discussion and interpretation of the data. APNM, the principal investigator, was responsible for planning, designing, analysis of the data and overall supervision of the work and final preparation of the manuscript. All authors read and approved the final manuscript.

\section{Authors' information}

Yingjie Yu, M.D.: Research Assistant Professor, Department of Internal Medicine and Veterans Affairs Medical Center, Wayne State University, Detroit, MI 48201, USA. E-mail: aa5142@wayne.edu.

Pratima Nangia-Makker, Ph.D.: Research Scientist, Department of Internal Medicine, Veterans Affairs Medical Center and Karmanos Cancer Institute, Wayne State University, Detroit, Ml 48201, USA. E-mail:

makkerp@karmanos.org.

Lulu Farhana, Ph.D.: Research Scientist, Department of Internal Medicine and Veterans Affairs Medical Center, Wayne State University, Detroit, Ml 48201, USA. E-mail: Ifarhana@med.wayne.edu.

Sindhu G. Rajendra, M.D. Visiting Scholar Department of Veterans Affairs Medical Center, Detroit, MI 48201, USA. E-mail: sindhu25raj@gmail.com. Edi Levi, M.D. Associat Professor, Department of Pathology, Veterans Affairs Medical Center, Detroit, Ml 48201, USA. E-mail: Edi.Levi@va.gov. Adhip P.N. Majumdar, Ph.D., D.Sc.: Professor and Senior Research Career Scientist, Department of Internal Medicine, Veterans Affairs Medical Center and Karmanos Cancer Institute, Wayne State University, Detroit, Ml 48201, USA. E-mail: a.majumdar@wayne.edu.

\section{Acknowledgments}

This study was supported by grants (APNM) from the NIH (AG014343) and the Department of Veteran Affairs (I101BX001927). The funders had no role in study design, data collection and analysis, decision to publish, or preparation of the manuscript.

\section{Author details}

'Department of Veterans Affairs Medical Center, 4646 John R, Detroit, MI 48201, USA. ${ }^{2}$ Karmanos Cancer Center, Detroit, Ml 48201, USA. ${ }^{3}$ Departments of Internal Medicine, Wayne State University, Detroit, Ml 48201, USA.

Received: 3 December 2014 Accepted: 22 April 2015

Published online: 01 May 2015

\section{References}

1. O'Connell MJ, Campbell ME, Goldberg RM, Grothey A, Seitz JF, Benedetti JK, et al. Survival following recurrence in stage II and III colon cancer: findings from the ACCENT data set. J Clin Oncol. 2008;26(14):2336-41.

2. Sanders MA, Majumdar AP. Colon cancer stem cells: implications in carcinogenesis. Front Biosci (Landmark Ed). 2011;16:1651-62.

3. Dean M, Fojo T, Bates S. Tumour stem cells and drug resistance. Nat Rev Cancer. 2005;5(4):275-84.

4. Houthuijzen JM, Daenen LG, Roodhart JM, Voest EE. The role of mesenchymal stem cells in anti-cancer drug resistance and tumour progression. Br J Cancer. 2012;106(12):1901-6.

5. Dalerba P, Dylla SJ, Park IK, Liu R, Wang X, Cho RW, et al. Phenotypic characterization of human colorectal cancer stem cells. Proc Natl Acad Sci U S A. 2007;104(24):10158-63.

6. Patel BB, Yu Y, Du J, Levi E, Phillip PA, Majumdar AP. Age-related increase in colorectal cancer stem cells in macroscopically normal mucosa of patients 
with adenomas: a risk factor for colon cancer. Biochem Biophys Res Commun. 2009:378(3):344-7.

7. Yu Y, Kanwar SS, Patel BB, Nautiyal J, Sarkar FH, Majumdar AP. Elimination of colon cancer stem-like cells by the combination of curcumin and FOLFOX. Transl Oncol. 2009;2(4):321-8.

8. Yu Y, Kanwar SS, Patel BB, Oh PS, Nautiyal J, Sarkar FH, et al. MicroRNA-21 induces stemness by downregulating transforming growth factor beta receptor 2 (TGFbetaR2) in colon cancer cells. Carcinogenesis. 2012;33(1):68-76.

9. Hutvagner $\mathrm{G}$, Zamore PD. A microRNA in a multiple-turnover RNAi enzyme complex. Science. 2002:297(5589):2056-60

10. Medina PP, Nolde M, Slack FJ. OncomiR addiction in an in vivo model of microRNA-21-induced pre-B-cell lymphoma. Nature. 2010;467(7311):86-90.

11. Ziyan W, Shuhua Y, Xiufang W, Xiaoyun L. MicroRNA-21 is involved in osteosarcoma cell invasion and migration. Med Oncol. 2011:28(4):1469-74.

12. Valeri N, Gasparini P, Braconi C, Paone A, Lovat F, Fabbri M, et al. MicroRNA-21 induces resistance to 5 -fluorouracil by down-regulating human DNA MutS homolog 2 (hMSH2). Proc Natl Acad Sci U S A. 2010;107(49):21098-103.

13. Nautiyal J, Du JH, Yu YJ, Kanwar SS, Levi E, Majumdar APN. EGFR regulation of colon cancer stem-like cells during aging and in response to the colonic carcinogen dimethylhydrazine. Am J Physiol-Gastrointestin Liver Physiol. 2012;302(7):G655-63.

14. Bandres E, Cubedo E, Agirre X, Malumbres R, Zarate R, Ramirez N, et al. Identification by Real-time PCR of 13 mature microRNAs differentially expressed in colorectal cancer and non-tumoral tissues. Mol Cancer. 2006;5:29.

15. Sachdeva M, Zhu S, Wu F, Wu H, Walia V, Kumar S, et al. p53 represses c-Myc through induction of the tumor suppressor miR-145. Proc Natl Acad Sci U S A. 2009:106(9):3207-12.

16. Akao Y, Nakagawa Y, Naoe T. MicroRNA-143 and -145 in colon cancer. DNA Cell Biol. 2007;26(5):311-20.

17. Yu Y, Sarkar FH, Majumdar AP. Down-regulation of miR-21 induces differentiation of chemoresistant colon cancer cells and enhances susceptibility to therapeutic regimens. Transl Oncol. 2013;6(2):180-6.

18. Kanwar SS, Yu Y, Nautiyal J, Patel BB, Majumdar AP. The Wnt/beta-catenin pathway regulates growth and maintenance of colonospheres. Mol Cancer. 2010;9:212.

19. Roy S, Yu Y, Padhye SB, Sarkar FH, Majumdar AP. Difluorinated-curcumin (CDF) restores PTEN expression in colon cancer cells by down-regulating miR-21. PLoS One. 2013;8(7), e68543.

20. Spizzo R, Nicoloso MS, Lupini L, Lu Y, Fogarty J, Rossi S, et al. miR-145 participates with TP53 in a death-promoting regulatory loop and targets estrogen receptor-alpha in human breast cancer cells. Cell Death Differ. 2010;17(2):246-54.

21. Suzuki HI, Yamagata K, Sugimoto K, Iwamoto T, Kato S, Miyazono K. Modulation of microRNA processing by p53. Nature. 2009;460(7254):529-33.

22. Kent OA, Chivukula RR, Mullendore M, Wentzel EA, Feldmann G, Lee KH, et al. Repression of the miR-143/145 cluster by oncogenic Ras initiates a tumor-promoting feed-forward pathway. Genes Dev. 2010;24(24):2754-9.

23. Hatley ME, Patrick DM, Garcia MR, Richardson JA, Bassel-Duby R, van Rooij E, et al. Modulation of K-Ras-dependent lung tumorigenesis by MicroRNA-21. Cancer Cell. 2010;18(3):282-93.

24. Ibrahim AF, Weirauch U, Thomas M, Grunweller A, Hartmann RK, Aigner A. MicroRNA replacement therapy for miR-145 and miR-33a is efficacious in a model of colon carcinoma. Cancer Res. 2011;71(15):5214-24.

25. Gotte M, Mohr C, Koo CY, Stock C, Vaske AK, Viola M, et al. miR-145dependent targeting of junctional adhesion molecule $\mathrm{A}$ and modulation of fascin expression are associated with reduced breast cancer cell motility and invasiveness. Oncogene. 2010;29(50):6569-80.

26. Sachdeva M, Mo YY. MicroRNA-145 suppresses cell invasion and metastasis by directly targeting mucin 1. Cancer Res. 2010;70(1):378-87.

27. Zhang J, Guo H, Zhang H, Wang H, Qian G, Fan X, et al. Putative tumor suppressor miR-145 inhibits colon cancer cell growth by targeting oncogene Friend leukemia virus integration 1 gene. Cancer. 2011;117(1):86-95.

28. Talotta F, Cimmino A, Matarazzo MR, Casalino L, De Vita G, D'Esposito M, et al. An autoregulatory loop mediated by miR-21 and PDCD4 controls the AP-1 activity in RAS transformation. Oncogene. 2009:28(1):73-84

29. Zhang Z, Zha Y, Hu W, Huang Z, Gao Z, Zang Y, et al. The auto-regulatory feedback loop of microRNA-21/Programmed cell death protein 4/Activation Protein-1 (miR-21/PDCD4/AP-1) as a driving force for hepatic fibrosis development. J Biol Chem. 2013;288(52):37082-93.

30. Kern HB, Niemeyer BF, Parrish JK, Kerr CA, Yaghi NK, Prescott JD, et al. Control of MicroRNA-21 expression in colorectal cancer cells by oncogenic epidermal growth factor/Ras signaling and Ets transcription factors. DNA Cell Biol. 2012;31(8):1403-11.

31. van Solingen C, Seghers L, Bijkerk R, Duijs JM, Roeten MK, van Oeveren-Rietdijk AM, et al. Antagomir-mediated silencing of endothelial cell specific microRNA-126 impairs ischemia-induced angiogenesis. J Cell Mol Med. 2009;13(8A):1577-85.

32. Patel BB, Gupta D, Elliott AA, Sengupta V, Yu Y, Majumdar AP. Curcumin targets FOLFOX-surviving colon cancer cells via inhibition of EGFRs and IGF-1R. Anticancer Res. 2010;30(2):319-25.

33. Xu H, Yu Y, Marciniak D, Rishi AK, Sarkar FH, Kucuk O, et al. Epidermal growth factor receptor (EGFR)-related protein inhibits multiple members of the EGFR family in colon and breast cancer cells. Mol Cancer Ther. 2005;4(3):435-42

34. Hu Y, Smyth GK. ELDA: extreme limiting dilution analysis for comparing depleted and enriched populations in stem cell and other assays. J Immunol Methods. 2009:347(1-2):70-8.

\section{Submit your next manuscript to BioMed Central and take full advantage of:}

- Convenient online submission

- Thorough peer review

- No space constraints or color figure charges

- Immediate publication on acceptance

- Inclusion in PubMed, CAS, Scopus and Google Scholar

- Research which is freely available for redistribution 